\title{
Several problems of exoplanetary orbits determination from radial velocity observations
}

\author{
Roman V. Baluev \\ Sobolev Astronomical Institute, Saint Petersburg State University, \\ Universitetskij prospekt 28, Petrodvorets, Saint Petersburg 198504, Russia. \\ email: roman@astro.spbu.ru
}

\begin{abstract}
Existing algorithms of analysis of radial velocity time series are improved for the purposes of extrasolar planets detection and characterizing. Three important effects are considered: the poorly known radial velocity jitter, periodic systematic errors, and statistical bias due to non-linearity of models. Mathematical tools to account for these effects are developed and applied to a number of real planetary systems. In particular, it is shown that two outer planets of HD37124 are likely trapped in the 2/1 resonance. The dwarf star GJ876 may host an extra, Neptune-mass, planet which is in resonance with two giant planets in this system.
\end{abstract}

Keywords. methods: data analysis, techniques: radial velocities, stars: planetary systems

\section{Introduction}

For now, more than 200 exoplanets orbiting main-sequence stars are known (Schneider 2007). Among the known planetary systems more than 20 contain two planets at least. Significant fraction of multiple planet systems possesses an interesting dynamics like mean-motion resonances and apsidal corotations. Still, the majority of planetary systems was discovered by radial velocity (RV) techniques.

Unfortunately, orbital parameters (and masses) in many planetary systems are still poorly determined, especially in multiple ones. The best-fitting orbital solutions for such systems may appear dynamically unstable in short timescales. In accordance with Goździewski et al. (2008), the stability constraint may be accounted for during data analysis, but sometimes this approach simply imposes a preferred result. Often, when recovering a chronology of orbital fits for a given planetary system we can note that parameters for subsequent fits may differ surprisingly largely.

The above reasoning makes us to assume that something is not accounted for during analysis of RV data. The main aim of this work is to discuss three important effects which could be this 'something'. They are (i) the poorly constrained RV jitter, (ii) periodic systematic errors, and (iii) statistical bias due to non-linearity of models. They are described in more details in Section 2. Section 3 describes briefly mathematical algorithms I used to account for these effects. Section 4 discusses preliminary results obtained for several planetary systems. Section 5 ends this contribution by conclusions. 


\section{Overview}

\subsection{Radial velocity jitter}

During analysis of RV data from planet search surveys, it is routinely assumed that the total variance $\sigma_{i}^{2}$ of an $i^{\text {th }} \mathrm{RV}$ measurement is a sum of the instrumental error variance $\sigma_{\text {mes }, i}^{2}$ and of the constant 'jitter' $\sigma_{\star}^{2} \equiv p$. In the astrophysical part, this jitter is inspired by various processes on the star leading to an apparent instability of its radial velocity. It also depends on the instrument, on the way of observation and its reduction to final radial velocity measurement. For example, the spectrum exposition as long as $20-30 \mathrm{~min}$ averages out apparent RV variations inspired by stellar oscillations (which have periods $\sim 5-10 \mathrm{~min}$ for solar-like stars). This decreases the astrophysical part of the RV jitter. However, the astrophysical jitter does not represent the only source of RV variations beyond the expected noise level. Many other sources like extra systematic RV errors lie in the instrument (but they may also depend on stellar properties).

It is very difficult to split the extra RV variability in parts originating from different physical sources. Moreover, this problem seems to be rather well separated from the problem of determination of planetary parameters. Due to this reasoning, I will use in this paper the generalized notion 'RV jitter' (or 'full RV jitter') as any short-term RV variability beyond the currently expected instrumental noise, for the current model of the RV curve. The full RV jitter consists of the astrophysical jitter (originating in the star), of the instrumental RV errors not accounted for in the current RV uncertainties (originating in the instrumentation and its nearest environment), and of the extra RV variations not accounted for in the current RV model. In Section 4 we will see that full RV jitters may be quite different for different observatories, as well their zero velocities are. Hence, when we perform a joint analysis of RV time series from different observatories, we should account for possible differences in RV jitters. Any undetected planets would also increase the effective jitter, but this increasing does not depend on an instrument.

Usually, the jitter is determined using empirical models based on stellar characteristics (Wright 2005). Unfortunately, this way of jitter estimation allows accuracies of only $\sim 50 \%$ or sometimes worse. Jitter of a few $\mathrm{m} / \mathrm{s}$ remains almost unconstrained a priori (in comparison with magnitudes of instrumental errors) and represents, in fact, an extra unknown parameter. Note, that statistical algorithms which are used usually for obtaining orbital fits require an input sequence of weights of measurements $\left(w_{i} \propto \sigma_{i}^{2}\right)$. Therefore, they assume multiplicative model $\sigma_{i}^{2}=\kappa / w_{i}$ and estimate implicitly the unspecified factor $\kappa$. There is no principle obstacles to modify these algorithms for the case of the square-additive model $\sigma_{i}^{2}=\sigma_{\text {mes }, i}^{2}+\sigma_{\star}^{2}$ with the last term being considered as a free parameter. Such extension may be done by transition from $\chi^{2}$-minimization to likelihood function maximization. Related mathematical questions are discussed in Section 3.

\subsection{Periodic instrumental errors}

Instrumental errors always contain a systematic part, along with a random one. Periodic or quasiperiodic systematic errors in radial velocities can significantly affect parameter estimations of also periodic planetary RV signals. In an extreme case, these systematic errors may be interpreted as Doppler signature of a planet. Fortunately, periods of instrumental systematic errors often may be identified a priori. For ground-based observations, they usually coincide with well-known periods like one year, one day (and one sidereal day), one month (either sidereal or synodic). Note that the timings for a given RV time series are usually well concentrated near some fixed sidereal time (in order to observe 
a given star near a fixed position on the sky). This concentration (usually within a one third part of the sidereal day) makes any diurnal periodicity approximately equivalent to an annual one. Hence, we are left with three prior periods only: 1 year $(\approx 365.25$ days, seasonic variation of observing conditions $), 1$ sidereal month $(\approx 27.32$ days, variation of the angular distance between the Moon and the star being observed) and 1 synodic month $(\approx 29.53$ days, variation of the Moon phase).

Section 4 will show that the annual errors of a few or even ten $\mathrm{m} / \mathrm{s}$ is a common phenomenon in current planet search surveys, rather than exception. During the symposium, I have got from several participants a number of explanations of sources for such systematic errors, which acted in datasets that I analysed. They are as follows:

(a) Inaccurate barycentric correction of radial velocities.

(b) Incomplete exclusion of telluric lines during spectrum analysis.

(c) Variations in spectral line profiles having not well-established nature.

We can see that the annual errors may originate from very different sources having either physical or mathematical nature.

\subsection{Non-linear effects}

It is well known that estimations of coefficients of a linear model, provided by minimization of a $\chi^{2}$ function, are unbiased (i.e., their expectations equal the true values of parameters). This property is important, because it allows us to hope that our estimations are related to the true values at all. Keplerian models are non-linear. In this case, the traditionally used $\chi^{2}$ minimization or likelihood maximization principles provide, strictly speaking, biased estimations. However, this bias tends to zero when the number of observations grows (see references in the work by Baluev (2008a)).

Nevertheless, for real radial velocity time series, the biasing of Keplerian parameters and jitter may become practically significant and may exceed respective uncertainties in several times. For instance, we can never get negative estimation of orbital eccentricity; thus we always overestimate eccentricity for a circular orbit and probably we systematically overestimate any non-zero eccentricity. This means that estimations of orbital eccentricities of exoplanets are probably biased to larger values. For multiple planet systems we can quite be left with less than 10 RV measurements per free parameter. With such lack of observations, we may get biases exceeding formal uncertainties essentially.

\section{Mathematical methods}

\subsection{Accounting for radial velocity jitter}

Evidently, RV jitter is not an usual fitting parameter and cannot be estimated during usual $\chi^{2}$ minimization. Information about the jitter may be extracted from the scattering of radial velocity measurements around best-fitting orbital solution. Mathematically, the jitter can be estimated using maximum likelihood principle. For $N$ Gaussian observations $v_{i}$, the doubled log-likelihood function may be written down as

$$
2 \ln \mathcal{L}(\theta, p)=-\sum_{i=1}^{N} \frac{\left(v_{i}-\mu\left(t_{i}, \theta\right)\right)^{2}}{\sigma_{i}^{2}(p)}-\sum_{i=1}^{N} \ln \sigma_{i}^{2}(p)+\text { const },
$$

where $\mu(t, \theta)$ is the fit model having $d$ 'usual' fitting parameters $\theta$ (planetary orbital elements, RV semi-amplitudes, etc.) and the RV uncertainty model $\sigma_{i}^{2}(p)=\sigma_{\text {mes }, i}^{2}+p$ 
introduces a 'variance' parameter - the jitter $p$. The first sum in the right hand side of eq. (3.1) represents the $\chi^{2}$ function $\dagger$ which depends on the usual parameters $\theta$ and on the jitter $p$. The second term depends on the jitter only. The joint estimation of the parameters $\theta$ and $p$ can be obtained in result of maximizing the full function (3.1). That is, the values of $\theta$ and $p$ corresponding to the maximum of $\mathcal{L}(\theta, p)$ should be adopted as the 'maximum-likelihood estimations'. The maximum value of the likelihood function itself measures the quality of the fit found. It is important that the resulting estimation of $p$ is automatically accounted for in the resulting estimations of $\theta$ (and vice versa).

To write down (3.1), we have assumed Gaussian distributions for RV errors. If this assumption is wrong, the function (3.1) is not a likelihood function and parameter estimations obtained during its maximization may be somewhat shifted with respect to the true maximum-likelihood estimations. For large datasets, however, the central limit theorem implies that the influence of non-Gaussian errors should tend to zero as $N \rightarrow \infty$. The non-gaussianity may affect the uncertainties of our estimations (by about $\mathcal{O}(1 / \sqrt{N})$ ) and their bias (by $\mathcal{O}(1 / N)$ ), but does not introduce a constant shift. These effects are calculated (till the first order) by Baluev (2008b) and are shown to be negligible or tolerable in usual practical situations.

\subsection{Bias reduction}

For the RV jitter, I applied an analytic way of bias reduction. Its first step is a built-in reduction achieved by slight modification of the function (3.1) as

$$
2 \ln \tilde{\mathcal{L}}(\theta, p)=-\frac{N}{N-d} \sum_{i=1}^{N} \frac{\left(v_{i}-\mu\left(t_{i}, \theta\right)\right)^{2}}{\sigma_{i}^{2}(p)}-\sum_{i=1}^{N} \ln \sigma_{i}^{2}(p) .
$$

This $\mathcal{O}(1 / N)$ modification normally allows to correct a large fraction of the bias in jitter. This decreases the cross-influence on estimations of parameters $\theta$ (remind that these estimations depend on the jitter). The rest of the first-order (i.e., $\mathcal{O}(1 / N)$ ) bias in $\sigma_{\star}^{2}$ can be calculated analytically as it is described by Baluev (2008b).

To correct the first-order bias in estimations, I used also the numerical algorithm proposed by Quenouille (1956). It is as follows:

(a) Calculate the basic (biased by $\mathcal{O}(1 / N)$ ) estimation $x$ of a given parameter $\xi$ from the full time series of $N$ observations.

(b) Construct $N$ reduced time series with $i^{\text {th }}(i=1,2, \ldots N)$ measurement omitted. Therefore, each reduced time series should consist of $N-1$ data points.

(c) Calculate $N$ new estimations $x_{i}^{\prime}(i=1,2, \ldots N)$ of $\xi$ by re-fitting with every of the reduced time series. The bias of $x_{i}^{\prime}$ will be about $\sim 1 /(N-1)$, hence these new estimations will be shifted with respect to $x$ by about $\sim(1 /(N-1)-1 / N)=\mathcal{O}\left(1 / N^{2}\right)$.

(d) Calculate the sum $b_{1}=\sum_{i=1}^{N}\left(x_{i}^{\prime}-x\right)$. The result $b_{1}=\mathcal{O}(1 / N)$ represents the firstorder bias of $x$. That is, the corrected estimation $x-b_{1}$ should be biased by $\mathcal{O}\left(1 / N^{2}\right)$ only.

The main advantage of this algorithm is that its implementation is model-independent and easy. This algorithm is valid for a wide variety of estimations (it is not restricted to, say, maximum-likelihood estimations only). Also, this algorithm does not require for the distribution of RV errors to be Gaussian (it is not restricted to any narrow family

$\dagger$ The $\chi^{2}$ random quantity is defined as the sum of $N$ standard (mean zero, variance one) independent Gaussian random quantities. For arbitrary values of $\theta$ and $p$, the first sum in (3.1) is not necessary a $\chi^{2}$ quantity. But the words ' $\chi^{2}$ function' denote (within this paper) the first sum in (3.1) as a function of parameters to be estimated. This should not confuse the reader. 
of distributions). Unfortunately, it is rather time-consuming because it requires making many fits of non-linear Keplerian models.

\subsection{Likelihood ratio periodograms}

Routinely used normalizations of the Lomb-Scargle periodogram assume fixed weights for observations and, hence, the multiplicative model for RV uncertainties. For planet searches, it is preferred to use some extension of such periodogram with a built-in estimation of the square-additive RV jitter. I used a direct extension of the least-squares periodogram $z_{3}(f)$ from (Baluev 2008a). This extension is based on the likelihood ratio statistic and is described in more details in (Baluev 2008b).

\subsection{Periodic systematic errors}

To account for possible annual errors in radial velocities, I added to RV models a simple harmonic term $A \cos (2 \pi(t-\tau) / 1 \mathrm{yr})$ of one year period. Usually, observations do not cover the full year but span a restricted observing season (always the same). Given the phase coverage $\sim 50 \%$, we may not care much about possible deviations of systematic errors from a sinuous curve.

\section{Preliminary results}

\subsection{Planetary systems with well-determined orbital solutions}

To test the above described algorithms of jitter estimation, it is worth applying them to a number of planetary systems with well-determined parameters and large numbers of observations. For this purpose, I have selected planetary systems around stars $51 \mathrm{Peg}$, 70 Vir, 14 Her, HD83443, HD69830, $\mu$ Ara and 55 Cnc. The respective maximumlikelihood solutions are given in the first seven records in Table 1. We can see that the presence of annual periodicity in data is a rather frequent (although, cases free from it are not seldom). In ELODIE, CORALIE, and HET data, the semi-amplitude of this periodicity may reach $10-15 \mathrm{~m} / \mathrm{s}$. Sometimes these periodicities produce clear peaks on periodograms and could be detected directly. In less bright examples, these peaks possess a high significance (given their frequency is known a priory), but may look unremarkable in comparison with the noise in a wide frequency range (see Fig. 1). On contrary, HARPS seems to be free from systematic errors. Data from Lick and Keck observatories show statistically significant annual periodicity in rare cases and with less semi-amplitude.

Addition of an annual term solves some inconsistencies between different surveys. For instance, a shallow linear RV trend detected by Butler et al. (2006) for $51 \mathrm{Peg}$, is not seen in the ELODIE data without accounting for the annual term with $A \sim 10 \mathrm{~m} / \mathrm{s}$.

We can clearly see that the best-fitting values of RV jitter for one and the same star can differ largely for different instruments. RV jitters for ELODIE and CORALIE usually exceed those for other spectrographs, even with annual errors taken into account. This may indicate some extra systematic errors of unclear nature. Vice versa, HARPS jitters are perfectly less than for other instruments. Data from Keck, Lick and AAT demonstrate intermediate cases. It is surprising that the jitter estimation of HJST data for 14 Her is definitely negative $\left(\sigma_{\star}^{2}<0\right)$, though no annual term was added to this dataset and the orbital solution is well constrained by data from other instruments. This indicates that 

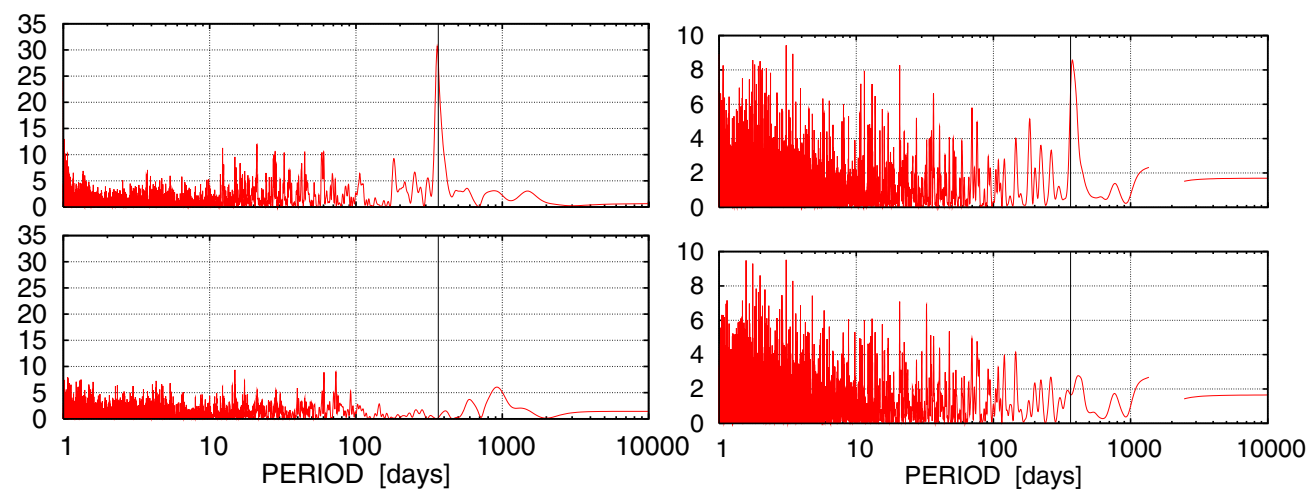

Figure 1. Left-Top: the modified likelihood ratio periodogram of RV residuals to the ELODIE radial velocities for the star 51 Pegasi. The RV variation induced by the planet $51 \mathrm{Peg} \mathrm{b}$ was included in the base RV model. Left-Bottom: a similar periodogram, but the annual term and the linear trend were included in the base RV model also. Right: similar periodgrams for the ELODIE radial velocities of 14 Herculis (base RV models: 'planet b + quadratic trend' for the top panel and 'planet $\mathrm{b}+$ quadratic trend + annual harmonic' for the bottom panel). Positions of the annual period are marked by extra vertical lines.

the RV uncertainties stated by Wittenmyer et al. (2007) for 14 Her overestimate real errors by about $20 \%$.

\subsection{Planetary system orbiting HD74156}

The period of the third planet in this system (recently announced based on HET observations, Bean et al. (2007)) is consistent with one year. The corresponding RV semiamplitude is only $12 \mathrm{~m} / \mathrm{s}$. This oscillation is opposite in phase to that of ELODIE data for the same star and is similar to the annual periodicity in HET data for 55 Cnc. Therefore, it is quite possible that the planet HD74156 d is a false-detection inspired by instrumental systematic errors. At least, parameter estimations of this putative planet may be highly distorted by the systematic errors.

\subsection{Planetary system orbiting $47 \mathrm{UMa}$}

Wittenmyer et al. (2007) could not clearly confirm existence of the second planet with a period of about 2500 days. Instead, they found an extra signal having semi-amplitude $10 \mathrm{~m} / \mathrm{s}$ and a very long period $\sim 8000$ days. In fact, this signal represents a shallow quadratic RV trend which is probably produced by a distant companion of poorly constrained period and mass. Addition of this parabolic trend to the model allowed to extract and confirm extra 2500 days periodicity of the planet 'c'. However, its semi-amplitude drops to $6-8 \mathrm{~m} / \mathrm{s}$, what implies the minimum mass $m_{c} \sin i_{c} \approx 0.5 M_{J \text { up }}$ only. 


\subsection{Planetary system orbiting HD11964}

The Extrasolar Planets Encyclopaedia by J. Schneider states that this system has two planets with periods of about 38 days (planet 'b') and 2000 days (planet 'c'), both discovered in 2005. However, there is no paper concerning the planet ' $\mathrm{b}$ ' in this Encyclopaedia. Butler et al. (2006) don't mention the 38 days planet, but report on the detection of the outer (2000 days) planet and refer to it as planet ' $b$ '. Likelihood periodogram of these data (with RV signal of the outer planet excluded) shows two almost equal and independent peaks near periods of 38 days and 365 days. In this case, the annual periodicity peak can be clearly seen on the periodogram. This annual periodicity should be accounted for in RV models in order to obtain more reliable estimations of the putative 38 days planet.

\subsection{Planetary system orbiting HD37124}

This system was a subject of many studies. The main problem is that the best-fitting orbital solution is far from dynamical stability due to a large eccentricity $e_{c} \approx 0.5$ of the outermost planet. It is possible to account for the stability requirement during data analysis (Goździewski et al. 2008). However, this implies too large loss in the fit quality. Moreover, these stable configurations occupy mainly boundaries of stability regions. This makes us to suppose that something is wrong with every of these orbital configurations.

Keck data alone yield an orbital fit not discussed in the discovery paper by Vogt et al. (2005). This fit has somewhat less period $P_{c}$ and larger period $P_{d}$, so that the outer planets become trapped into $2 / 1$ mean-motion resonance. This fit can be easily missed by a fitting algorithm due to larger values of r.m.s and $\chi^{2}$ (or lower likelihood $\mathcal{L}$ ). However, if we enlarge fitting model by including some extra free terms (linear trend or annual periodicity), the picture becomes opposite. The main advantage of the resonant fit is that both orbital eccentricities become ill-determined and strongly biased to larger values. Therefore, it becomes possible to find a stable solution (having moderate eccentricities) with tolerable decreasing in fit quality.

Table 1 contains biased maximum-likelihood estimations of parameters for this system. Quenouille's bias reduction decreases eccentricities to ill-determined values $e_{c}=0.3 \pm 0.2$ and $e_{d}=0.3 \pm 0.1$. Hence, the most likely structure of this system is a pair of resonant $(2 / 1)$ outer planets significantly perturbed by the inner (non-resonant) one.

\subsection{Planetary system orbiting GJ876}

Rivera et al. (2005) mentioned that periodogram of RV residuals to the best-fitting dynamical model for this three-planet system is not clean but contains at least three uncomfortable peaks at periods about 9,13 and 125 days. I have re-fitted their radial velocities with free annual term added to Keck RVs and plotted a periodogram of resulting residuals. The 125 days peak rised up whereas other peaks retained at low levels. This 125 days periodicity may be interpreted as a fourth, Neptune-mass, planet in the system (although significantly negative value of Keck jitter estimation inspires some doubts and requires further studies). Dynamical behaviour of such four-planet system is remarkable due to the triple resonance $4 / 2 / 1$ of outer planets. 
Table 1. Revised data for several planetary systems.

\begin{tabular}{|c|c|c|c|c|c|c|c|c|c|c|}
\hline Planet & & $P$ [days $]$ & $\tilde{K}[\mathrm{~m} / \mathrm{s}]$ & $l_{0}\left[{ }^{o}\right]$ & $e$ & $\omega\left[{ }^{o}\right]$ & Instr & $A[\mathrm{~m} / \mathrm{s}]$ & $\tau$ [days] & $\sigma_{\star}[\mathrm{m} / \mathrm{s}]^{1}$ \\
\hline $51 \mathrm{Peg}^{3} \mathrm{~b}$ & & $4.230791(31)$ & $55.80(48)$ & $59.74(60)$ & $0.0089(88)$ & $31(53)$ & $\begin{array}{l}\text { ELD } \\
\text { LCK }\end{array}$ & $11.2(1.2)$ & $364.7(9.3)$ & $\begin{array}{c}6.56(83) \\
0.5(2.8)\end{array}$ \\
\hline $70 \mathrm{Vir} b$ & & $116.6901(44$ & $288.8(1.0)$ & $79.48(18)$ & $0.4009(28)$ & $359.16(44)$ & $\begin{array}{l}\text { ELD } \\
\text { LCK }\end{array}$ & $5.1(1.6)$ & $315(16)$ & $\begin{array}{l}-2.8(1.8)^{2} \\
3.58(79)\end{array}$ \\
\hline $14 \mathrm{Her}^{3} \mathrm{~b}$ & & $1771.3(2.4)$ & $83.61(45)$ & $306.23(42)$ & $0.3683(45)$ & $21.88(85)$ & $\begin{array}{l}\text { ELD } \\
\text { LCK } \\
\text { HJS }\end{array}$ & $6.4(1.6)$ & $185(12)$ & $\begin{array}{l}6.45(96) \\
1.23(45) \\
-4.44(86)^{2}\end{array}$ \\
\hline HD $83443^{3}$ & $3 \mathrm{~b}$ & $2.985746(36$ & $57.12(60)$ & $144.0(1.7)$ & $0.013(10)$ & $157(48)$ & $\begin{array}{l}\text { COR } \\
\mathrm{AAT} \\
\mathrm{KCK}\end{array}$ & $5.8(1.4)$ & $99.7(9.5)$ & $\begin{array}{l}6.40(48) \\
6.0(1.7) \\
2.70(58)\end{array}$ \\
\hline HD 69830 & $\begin{array}{l}\mathrm{b} \\
\mathrm{c} \\
\mathrm{d}\end{array}$ & $\begin{array}{l}8.6674(23) \\
31.564(41) \\
196.6(2.3)\end{array}$ & $\begin{array}{l}3.49(15) \\
2.64(18) \\
2.20(20)\end{array}$ & $\begin{array}{l}224.6(6.5) \\
264.6(8.5) \\
289(13)\end{array}$ & $\begin{array}{l}0.101(42) \\
0.126(62) \\
0.069(84)\end{array}$ & $\begin{array}{l}340(24) \\
220(35) \\
225(65)\end{array}$ & HRP & & & $0.22(26)$ \\
\hline $\begin{array}{r}\mu \text { Ara } \\
\text { c } \\
\text { d } \\
\text { e }\end{array}$ & & $\begin{array}{l}644.7(1.4) \\
3900(700) \\
9.6388(23) \\
310.0(1.2)\end{array}$ & $\begin{array}{l}36.84(57) \\
21.5(2.3) \\
2.88(22) \\
15.1(1.1)\end{array}$ & $\begin{array}{l}18.22(63) \\
70(11) \\
185.5(5.3) \\
166.8(2.6)\end{array}$ & $\begin{array}{l}0.101(30) \\
0.070(34) \\
0.172(77) \\
0.083(19)\end{array}$ & $\begin{array}{l}33(14) \\
100(90) \\
212(26) \\
195(14)\end{array}$ & $\begin{array}{l}\text { COR } \\
\text { AAT } \\
\text { HRP }\end{array}$ & & & $\begin{array}{l}5.67(95) \\
2.37(25) \\
1.52(15)\end{array}$ \\
\hline $\begin{array}{l}55 \mathrm{Cnc}^{4} \\
90(\text { fixed })\end{array}$ & $\begin{array}{l}\mathrm{b} \\
\mathrm{c} \\
\mathrm{d} \\
\mathrm{e}\end{array}$ & $\begin{array}{l}14.6491(15) \\
44.472(50) \\
4870(120) \\
2.79579(13)\end{array}$ & $\begin{array}{l}71.78(64) \\
9.84(70) \\
46.09(97) \\
4.93(68)\end{array}$ & $\begin{array}{l}149.3(1.0) \\
24.8(5.9) \\
86.2(2.6) \\
61.9(8.4)\end{array}$ & $\begin{array}{l}0.0058(99) \\
0.186(23) \\
0.235(30) \\
0.26(14)\end{array}$ & $\begin{array}{l}116(87) \\
39(11) \\
208.3(7.5) \\
186(30)\end{array}$ & $\begin{array}{l}\text { ELD } \\
\text { LCK } \\
\text { HET }\end{array}$ & $\begin{array}{l}10.4(3.4) \\
13.6(2.5)\end{array}$ & $\begin{array}{l}86(19) \\
229.7(7.8)\end{array}$ & $\begin{array}{l}8.9(1.5) \\
5.18(50) \\
5.87(77)\end{array}$ \\
\hline HD74156 & $\begin{array}{l}\mathrm{b} \\
\mathrm{c}\end{array}$ & $\begin{array}{l}51.6413(26) \\
2452.3(9.3)\end{array}$ & $\begin{array}{l}87.7(1.1) \\
105.4(2.2)\end{array}$ & $\begin{array}{l}79.39(60) \\
201.87(82)\end{array}$ & $\begin{array}{r}0.6333(73) \\
0.3901(10)\end{array}$ & $\left\{\begin{array}{l}176.58(89) \\
267.8(2.4)\end{array}\right.$ & $\begin{array}{l}\text { ELD } \\
\text { COR } \\
\text { HET }\end{array}$ & $\begin{array}{l}21.0(5.8) \\
12.1(1.7)\end{array}$ & $\begin{array}{l}31.1(7.3) \\
) 255.5(8.0)\end{array}$ & $\begin{array}{l}-4.7(2.2)^{2} \\
11.6(1.9) \\
5.74(54)\end{array}$ \\
\hline $47 \mathrm{UMa}^{3}$ & $\begin{array}{l}\mathrm{b} \\
\mathrm{c}\end{array}$ & $\begin{array}{l}1073.7(1.5) \\
2520(110)\end{array}$ & $\begin{array}{l}49.91(97) \\
7.38(89)\end{array}$ & $\begin{array}{l}28.0(1.0) \\
77.0(7.8)\end{array}$ & $\begin{array}{l}0.018(17) \\
0(\text { fixed })\end{array}$ & $\begin{array}{l}108(50) \\
0(\text { fixed })\end{array}$ & $\begin{array}{l}\text { ELD } \\
\text { LCK } \\
\text { HJS } \\
\text { HET }\end{array}$ & $\begin{array}{l}10.9(3.7) \\
5.2(1.4)\end{array}$ & $\begin{array}{l}98(19) \\
207(14)\end{array}$ & $\begin{array}{l}7.7(1.6) \\
2.96(78) \\
4.8(1.6) \\
4.66(44)\end{array}$ \\
\hline HD11964 & $\begin{array}{l}\mathrm{b} \\
\mathrm{c}\end{array}$ & $\begin{array}{l}38.002(59) \\
1893(40)\end{array}$ & $\begin{array}{l}3.73(56) \\
10.21(84)\end{array}$ & $\begin{array}{l}86.5(9.6) \\
300.2(3.8)\end{array}$ & $\begin{array}{l}0 \text { (fixed) } \\
0.051(63)\end{array}$ & $\begin{array}{l}0 \text { (fixed) } \\
184(78)\end{array}$ & $\mathrm{KCK}$ & $3.37(51)$ & $16(17)$ & $2.64(36)$ \\
\hline HD 37124 & $\begin{array}{l}\mathrm{b} \\
\mathrm{c} \\
\mathrm{d}\end{array}$ & $\begin{array}{l}154.53(12) \\
1777(24) \\
902.3(8.7)\end{array}$ & $\begin{array}{l}27.43(73) \\
15.2(1.0) \\
15.1(1.1)\end{array}$ & $\begin{array}{l}308.6(3.0) \\
250.5(6.0) \\
119.9(6.5)\end{array}$ & $\left\{\begin{array}{l}0.108(29) \\
0.563(56) \\
0.441(65)\end{array}\right.$ & $\begin{array}{l}134(14) \\
251.9(6.6) \\
276(13)\end{array}$ & $\begin{array}{l}\text { ELD } \\
\text { COR } \\
\text { KCK }\end{array}$ & $\begin{array}{l}17.5(3.5) \\
4.5(1.4)\end{array}$ & $\begin{array}{l}68(20) \\
122(19)\end{array}$ & $\begin{array}{l}7.4(2.3) \\
12.3(4.2) \\
1.24(71)\end{array}$ \\
\hline $\begin{array}{l}\text { GJ } 876^{4} \\
53.9(3.0)\end{array}$ & $\begin{array}{l}\mathrm{b} \\
\mathrm{c} \\
\mathrm{d}\end{array}$ & $\begin{array}{l}60.815(19) \\
30.482(19) \\
1.937836(47\end{array}$ & $\begin{array}{l}213.43(56) \\
85.18(65) \\
6.44(50)\end{array}$ & $\begin{array}{l}203.65(22) \\
42.1(1.0) \\
347.3(6.3)\end{array}$ & $\begin{array}{l}0.0339(30) \\
0.2521(55) \\
0(\text { fixed })\end{array}$ & $\begin{array}{l}5.1(4.4) \\
3.85(95) \\
0(\text { fixed })\end{array}$ & $\begin{array}{l}\mathrm{KCK} \\
\mathrm{LCK}\end{array}$ & $4.44(81)$ & $284.7(8$ & $\begin{array}{l}-1.13(76)^{2} \\
-12.6(5.0)^{2}\end{array}$ \\
\hline $\begin{array}{l}\text { GJ } 876^{4} \\
53.8(2.3)\end{array}$ & $\begin{array}{l}\mathrm{b} \\
\mathrm{c} \\
\mathrm{d} \\
\mathrm{e}\end{array}$ & $\begin{array}{l}60.801(17) \\
30.491(15) \\
1.937826(42 \\
125.6(1.1)\end{array}$ & $\begin{array}{l}213.51(50) \\
84.81(56) \\
6.39(44) \\
3.46(45)\end{array}$ & $\begin{array}{c}203.58(18) \\
42.12(94) \\
348.6(5.3) \\
305.5(5.9)\end{array}$ & $\begin{array}{l}0.0385(26) \\
0.2520(43) \\
0(\text { fixed }) \\
0.095(24)\end{array}$ & $\begin{array}{l}2.9(4.0) \\
4.20(92) \\
0(\text { fixed }) \\
209(15)\end{array}$ & $\begin{array}{l}\mathrm{KCK} \\
\mathrm{LCK}\end{array}$ & $4.81(71)$ & $282.4(6$ & $\begin{array}{l}-2.32(25)^{2} \\
-11.9(5.9)^{2}\end{array}$ \\
\hline
\end{tabular}

\section{Notes:}

Orbital parameters are the usual Keplerian elements except for the modified semi-amplitude $\tilde{K}=K \sqrt{1-e^{2}}$. Mean longitudes $l_{0}$ and values of $\tau$ (and all other parameters for dynamical fits) are given for the epoch $J D=2453000$ for the stars HD69830, $\mu$ Ara, HD74156, HD11964, and $J D=2451000$ for other stars. The quantities $A, \tau, \sigma_{\star}$ are defined in the text. No bias correction applied here, except for analytic bias corrections of jitter estimations.

${ }^{1}$ Uncertainties of jitter estimations are calculated in the asymptotic Gaussian approximation as $\delta=\varepsilon /\left(2 \sigma_{\star}\right)$, where $\varepsilon$ is the uncertainty of the estimation of $p=\sigma_{\star}^{2}\left(\varepsilon\right.$ was estimated simultaneously with $p$ ). When $\sigma_{\star}$ is comparable with $\delta$, its distribution is far from Gaussian. Then it is necessary to return back to the quantity $p=\sigma_{\star}^{2}$ (not affected by the degeneracy) and to its uncertainty $\varepsilon=2 \delta \sigma_{\star}$

${ }^{2}$ Negative values of $\sigma_{\star}$ reflect symbolically that the corresponding estimations of $\sigma_{\star}^{2}$ are negative.

3 A linear/quadratic trend was included in the model.

${ }^{4}$ Dynamical co-planar fits (i.e., mutual gravitational interactions of planets are accounted for, all orbital planes are assumed co-planar). Fitted orbital inclinations of the systems (in degrees) are shown in the first column.

${ }^{5} \mathrm{ELD}=\mathrm{ELODIE}, \mathrm{COR}=\mathrm{CORALIE}, \mathrm{HRP}=\mathrm{HARPS}, \mathrm{LCK}=$ Lick Observatory, KCK = Keck Telescope, 

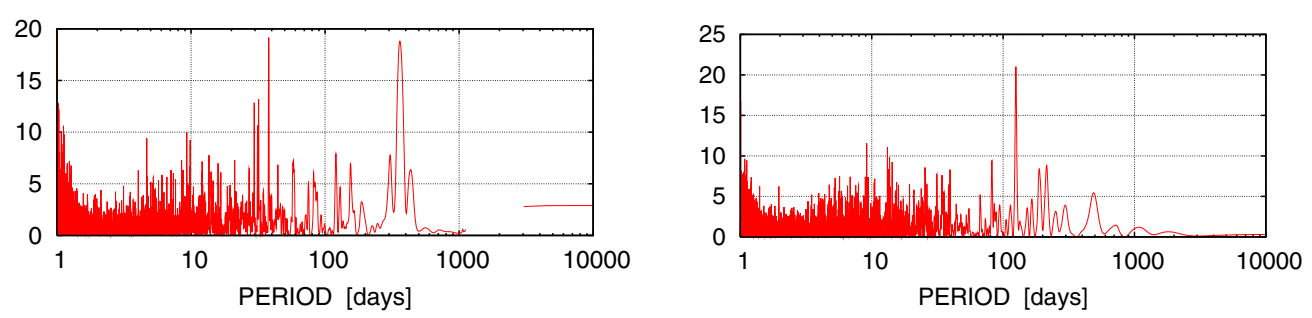

Figure 2. Left: periodogram of RV residuals for the star HD11964 with 2000-day planet included in the base model. Right: the periodogram of RV residuals for GJ876 to the best-fitting base model (dynamical three-planet RV curve + annual periodicity).

\section{Conclusions}

In this contribution, existing algorithms of analysis of radial velocity time series are improved for the purposes of detecting and characterizing extrasolar planets. A maximumlikelihood estimation of RV jitters is built in the usual RV curve fitting algorithms. Resulting algorithms often allow much higher accuracies than the jitter estimation based on empirical dependencies on stellar activity. It is shown that often the data from planet search radial velocity surveys suffer from periodic annual systematic errors. These errors should be taken into account during data analysis. Mathematical tools for statistical bias correction are proposed. Their usage is important mainly for multiple planet systems.

Application of improved algorithms allows to revise orbital solutions for a number of planetary systems. Recently announced planet HD74156 d may be a false-detection due to the annual systematic errors. The second planet orbiting $47 \mathrm{UMa}$ does exist but has a smaller mass than it was estimated initially. Two outer planets around HD37124 are probably trapped in the 2/1 resonance. Planetary system around GJ876 may contain a fourth planet 'e' orbiting in resonances $2 / 1$ and 4/1 with known giant planets GJ876 b,c.

\section{Acknowledgements}

I would like to thank Drs. V.V. Orlov and K.V. Kholshevnikov for critical reading of this paper, useful suggestions, and linguistic corrections. Dr S. Ferraz-Mello is thanked for fruitful suggestions concerning terminology used in the paper. I am grateful to Drs. M. Mayor, J.T. Wright, and A. Quirrenbach for detailed discussions of possible sources of annual RV errors in present planet search surveys. This work was supported by the Russian Foundation for Basic Research (Grants 05-02-17408, 06-02-16795) and by the President Grant NS-4929.2006.2 for the state support of leading scientific schools.

\section{References}

Baluev, R. V. 2008a, Mon. Not. R. Astron. Soc., (Online Early), doi:101111/j.13652966.2008.12689.x

Baluev, R. V. 2008b, Mon. Not. R. Astron. Soc., submitted (arXiv/astro-ph: 0712.3862)

Bean, J. L., McArthur, B. E., Benedict, G. F., \& Armstrong, A. 2007, Astroph. Journ., 672, 1202 
Butler, R. P., Wright, J. T., Marcy, G. W., Fischer, D. A., Vogt, S. S., Tinney, C. G., Jones, H. R. A., Carter, B. D., Johnson, J. A., McCarthy, C., \& Penny, A. J. 2006, Astroph. Journ., 646,505

Goździewski, K., Migaszewski, C. \& Musieliński, A. 2008, these proceedings

Quenouille, M. H. 1956, Biometrika, 43, 353

Rivera, E. J., Lissauer, J. J., Butler, R. P., Marcy, G. W., Vogt, S. S., Fischer, D. A., Brown, T. M., Laughlin, G., \& Henry, G. W. 2005, Astroph. Journ., 634, 625

Schneider, J. 2007, http://exoplanet.eu, The Extrasolar Planets Encyclopaedia

Vogt, S. S., Butler, R. P., Marcy, G. W., Fischer, D. A., Henry, G. W., Laughlin, G., Wright, J. T., \& Johnson, J. A. 2005, Astroph. Journ., 632, 638

Wittenmyer, R. A., Endl M., \& Cochran, W. D. 2007, Astroph. Journ., 654, 625

Wright, J. T. 2005, Pub. Astr. Soc. Pacific, 117, 657 